\title{
Co

\section{Mortality benefit from unrestricted access to clopidogrel: Too good to be true?}

\author{
Samy Suissa PhD
}

$\infty$

See related article page $4 \mathrm{I} 3$

$\mathrm{T}$ he use of clopidogrel, a thienopyridine antiplatelet agent, at the time of percutaneous coronary intervention has been generally shown in randomized controlled trials to reduce the risk of thrombosis and related complications. Clinicians are faced with questions about the optimal use of clopidogrel, including the dose and timing of administration. Thus, recent studies have evaluated whether and when to use a loading dose before percutaneous coronary intervention; whether a loading dose should be followed by a treatment course until the intervention is performed; whether clopidogrel should be used in the 2-4 weeks following the intervention; and whether the longterm use of clopidogrel is beneficial. The randomized trials conducted to date to address these questions are faced with complex methodologies because these trials involve multiple and sequential interventions, and they have received some criticism..$^{1-3}$ In Canada, because of the expense of this drug and its potential for misuse, various provincial formularies have placed it on the restricted-access list. As with all drugs on this list, we need to evaluate whether this restriction (special authorization is required for prescription) hinders access and leads to harm by underuse. In the absence of randomized controlled trials, observational studies can be useful tools to address such questions.

The observational study by Sheehy and colleagues in this issue of $C M A J$ addresses this intricate issue at the population level by evaluating whether the authorization process in Quebec had an effect on patients' filling of clopidogrel prescriptions and a subsequent effect on all-cause mortality. ${ }^{4}$ This study involved a cohort of over 13 ooo patients who were identified from Régie de l'assurance maladie du Québec (RAMQ) databases. These patients underwent percutaneous coronary intervention between January 2000 and December 2004 and had received a prescription for a nonrestricted cardiovascular drug after the intervention. The authors grouped the patients as follows: those who filled a clopidogrel prescription at the same time that they filled the nonrestricted cardiovascular drug prescription, those who filled a clopidogrel prescription with a delay after filling the nonrestrictive cardiovascular drug prescription and those who did not fill a clopidogrel prescription during the I-year follow-up. Compared with the Io 918 patients who filled a clopidogrel pre-

\section{Key points of the article}

- It is unknown whether restricting access to certain prescription drugs, such as clopidogrel, leads to harm by underuse.

- Observational database studies can be useful to rapidly address such questions.

- Immortal time bias artificially inflates mortality among nonusers of the drug being studied.

- Bias creates an apparent mortality benefit from unrestricted access to prescription drugs.

scription without delay, the I-year all-cause mortality was $70 \%$ higher among the 1565 patients who did not fill a clopidogrel prescription and $35 \%$ higher among the II 80 patients who filled a clopidogrel prescription with a delay.

This study suggests that the need for special authorization may have impeded patients' filling of prescriptions, because $20 \%$ of patients either had a delay before filling or did not fill a clopidogrel prescription. This study also suggests that prescribing and receiving clopidogrel without delay after percutaneous coronary intervention could reduce the number of deaths by about $33 \%$ despite an adherence rate of only $40 \%$. Although this finding implies that unrestricted access to this drug could have saved lives, the authors fittingly discuss potential sources of bias that are common to observational studies, including immortal time, confounding and information bias.

In cohort studies, immortal time is a period during followup when, by definition of the study groups, no deaths can occur. Immortal time bias results from misclassifying these periods in the analysis. ${ }^{5,6}$ There are 2 immortal time periods in the study by Sheehy and colleagues. The first is the time between the date of discharge after percutaneous coronary intervention and the date that the patient filled his or her first prescription for a nonrestricted cardiovascular drug (Figure I). By definition of this group, no deaths could have occurred during this time. The authors report some variability in the

Samy Suissa is with the Division of Clinical Epidemiology, Royal Victoria Hospital, McGill University Health Centre and the Department of Epidemiology and Biostatistics, McGill University, Montréal, Que. 
length of this period, with a mean length of 6.9 days among those who did not fill a clopidogrel prescription, I.6 days among those who filled a clopidogrel prescription with a delay and 3.I days among those who filled a clopidogrel prescription without a delay. Although these differences may not appear to be large, the resulting bias may be important because thrombotic risk is highest early after stent insertion. This bias could have easily been eliminated simply by taking the date that each patient first filled a nonrestricted cardiovascular drug prescription as time zero in the survival analysis, which would also have allowed consideration of the duration of this period as a covariable in the adjusted analysis.

The second, more complex, source of immortal time arises from the delay in filling clopidogrel prescriptions (Figure I). Compared with patients who filled both prescriptions at the same time, patients with a delay could not have died between when they filled a nonrestricted cardiovascular drug prescription and a clopidogrel prescription. Those who died during this period were necessarily all grouped with the patients who never filled a clopidogrel prescription. The impact of this immortal time is seen in the Kaplan-Meier curves, which show that during the first 30 days of follow-up only I death occurred among those who filled their clopidogrel prescription with a delay, compared with about 25 deaths among those who did not fill a clopidogrel prescription. Thus, this

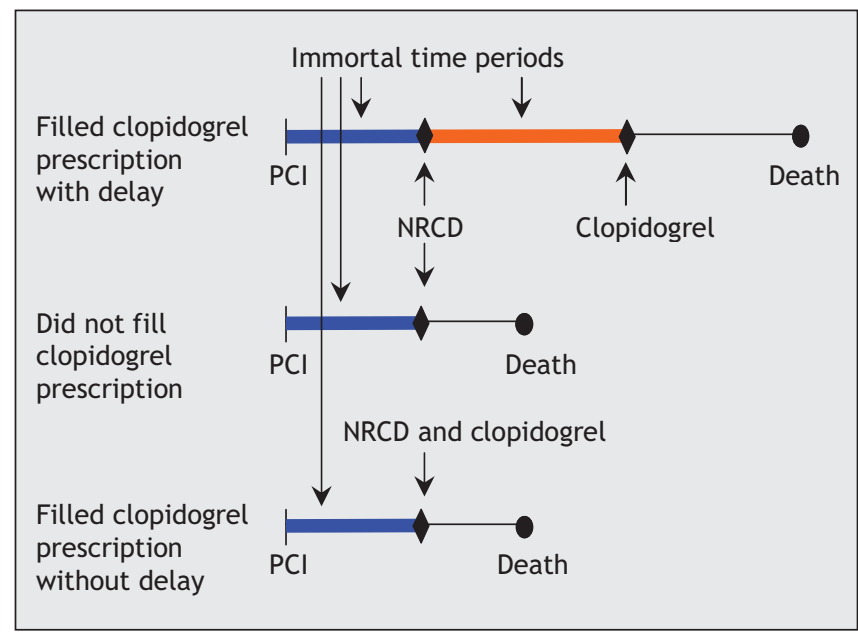

Figure 1: Immortal time in the study by Sheehy and colleagues of restrictive access to clopidogrel. ${ }^{4}$ Patients were grouped as follows: those with a delay of at least 1 day between filling a nonrestrictive cardiovascular drug (NRCD) prescription and a clopidogrel prescription; those who did not fill a clopidogrel prescription despite filling a nonrestricted cardiovascular drug prescription; and those who filled both prescriptions without a delay. The time between cohort entry (percutaneous coronary intervention $[\mathrm{PCI}]$ ) and the first nonrestricted cardiovascular drug prescription (blue line) is immortal for all 3 groups because the patients must have been alive during these periods to have filled their prescriptions. The time between the nonrestricted cardiovascular drug and clopidogrel prescriptions (orange line) is also immortal for those with a delay in filling a clopidogrel prescription. Patients who died during this period were necessarily grouped with those who did not fill a clopidogrel prescription.

\section{Immortal time bias may be present if:}

- It is an observational study (usually cohort studies).

- The drug exposure changes over time.

- The data analysis does not properly account for the changes in drug exposure over time.

- A surprisingly tremendous drug benefit is reported.

- The result is hard to believe or too good to be true (it probably isn't true).

bias generates artificial protection against death in the group with a delay and makes the delay appear protective.

The most challenging group, however, is that which includes patients who did not fill a clopidogrel prescription during the I-year follow-up period. This group includes patients who died after they filled a prescription for a nonrestricted cardiovascular drug but before they filled a clopidogrel prescription. Whether patients are included in this group depends on the length of the follow-up period. For example, if the authors had selected a 2-year follow-up period instead of a I-year period, some patients from the group who did not fill a clopidogrel prescription during the first year may be grouped with the patients who filled a clopidogrel prescription with a delay (if they filled their clopidogrel prescription during the second year). Assuming that this applies to onethird of the patients in the group who did not fill a clopidogrel prescription, the I-year survival among patients in the group who filled a clopidogrel prescription with a delay would then be equal to that of the group without delay. A study design in which the inclusion of patients in an exposure group depends on the length of follow-up, however unreasonable, is clearly unsound. Thus, the analysis that compares patients who filled a clopidogrel prescription without delay to all other patients, irrespective of whether or when they received clopidogrel, is free of this second source of immortal time bias. However, this analysis, which produces a hazard ratio of $\mathrm{I} .55$, is subject to confounding bias.

The issue of confounding bias is fundamental when the benefit of a drug (in this case the early use of clopidogrel to prevent death) is assessed in an observational study. Sheehy and colleagues adjusted all comparisons for differences in several covariables, including comorbidity (measured by a chronic disease score based on drug use in the year before percutaneous coronary intervention). Although this score predicts mortality ( $\mathrm{I} 4 \%$ increase for every unit of the score), it may have been measured at a too distant time. Patients who did not fill a clopidogrel prescription had a mean hospital stay of 15.6 days after percutaneous coronary intervention, compared with 9.2 days for patients who filled a clopidogrel prescription without a delay. Thus, these patients were likely sicker at the time of discharge. (Incidentally, these figures are surprisingly high because current practice is more in line with hospital stays of 2-3 days.) More up-to-date information on confounders that are predictive of mortality after percutaneous coronary intervention could have been obtained by taking advantage of the large number of discharge diagnoses and procedures available in the RAMQ databases, which also 
include data on complications at discharge, bleeding or other containdications for clopidogrel use. Another important potential confounder that could have been considered in the study by Sheehy and colleagues was the actual nonrestricted cardiovascular drug that was used to define the cohort. Although clopidogrel may be taken at the same time as lipidlowering, antihypertensive or oral hypoglycemic agents, physicians may be reluctant to prescribe clopidogrel with warfarin, particularly if acetylsalicylic acid is also being taken. Thus, a consideration in the analysis of the type of nonrestricted cardiovascular drug prescribed, perhaps by stratification, would allow tighter adjustment for confounding by indication and contraindication.

Finally, the absence of key data in the databases could have led to information bias. Indeed, the RAMQ databases do not permit one to verify whether the delay or absence of clopidogrel dispensing was because patients received a supply of clopidogrel at discharge or because they were noncompliant and did not fill a written and authorized prescription, which would be independent of the restrictive-access policy. Moreover, although the authors excluded patients who received outpatient prescriptions for clopidogrel in the year before percutaneous coronary intervention, the databases do not contain information about whether a loading dose or a pretreatment course was given to these patients. Finally, with hospital stays of more than 15 days after percutaneous coronary intervention, very sick patients may have received the 2week version of clopidogrel treatment while in hospital and none thereafter, which could have introduced further bias from misclassification.

The study by Sheehy and colleagues suggests that a delay, purportedly from a restrictive-access policy, in clopidogrel use after percutaneous coronary intervention may have led to an increase in mortality in Quebec. However, the study's observational design, lack of important information and various biases make this assertion doubtful. Whereas randomized controlled trials have demonstrated the benefit of clopidogrel on major cardiac outcomes, the suggestions from this observational study that unrestricted access to clopidogrel would have led to its earlier use after percutaneous coronary intervention and that it would reduce the number of all-cause deaths by one-third may simply be too good to be true. Further research, including well-designed randomized controlled trials and meticulously analyzed observational studies, is necessary before we can put forward such strong assertions about policies for public prescription drug management and their potential impact on public health.

Competing interests: Samy Suissa has served as an advisory board member and has received consultancy fees from Sanofi-Aventis (for Lantus and leflunomide but not for clopidogrel) and from Bristol-Myers Squibb (for Abatacept).

Acknowledgements: Samy Suissa is the recipient of a Distinguished Investigator award from the Canadian Institutes of Health Research.

\section{REFERENCES}

I. Cannon C. Clopidogrel and percutaneous coronary intervention. Lancet 2002;359:169.

2. Choudhury RP. Clopidogrel and percutaneous coronary interventions [letter]. JAMA 2003;289:1925.

3. Bridges JFP. Clopidogrel and percutaneous coronary interventions [letter]. JAMA 2003;289:1925-6.

4. Sheehy O, LeLorier J, Rinfret S. Restrictive access to clopidogrel and mortality following coronary stent implantation. CMAJ 2008;178:4I3-20.

5. Suissa S. Immortal time bias in observational studies of drug effects. Pharmacoepidemiol Drug Saf 2007; 6:24I-9.

6. Suissa S. Immortal time bias in pharmacoepidemiology. Am J Epid. PMID: I8056625. Epub 2007 Dec 3 ahead of print.

Correspondence to: Dr. Samy Suissa, Pharmacoepidemiology

Research Unit, McGill University, Ross 4.29, Royal Victoria

Hospital, 687 Pine Ave. W, Montréal QC H3A IAI;

fax 5I4 843-I493; samy.suissa@clinepi.mcgill.ca

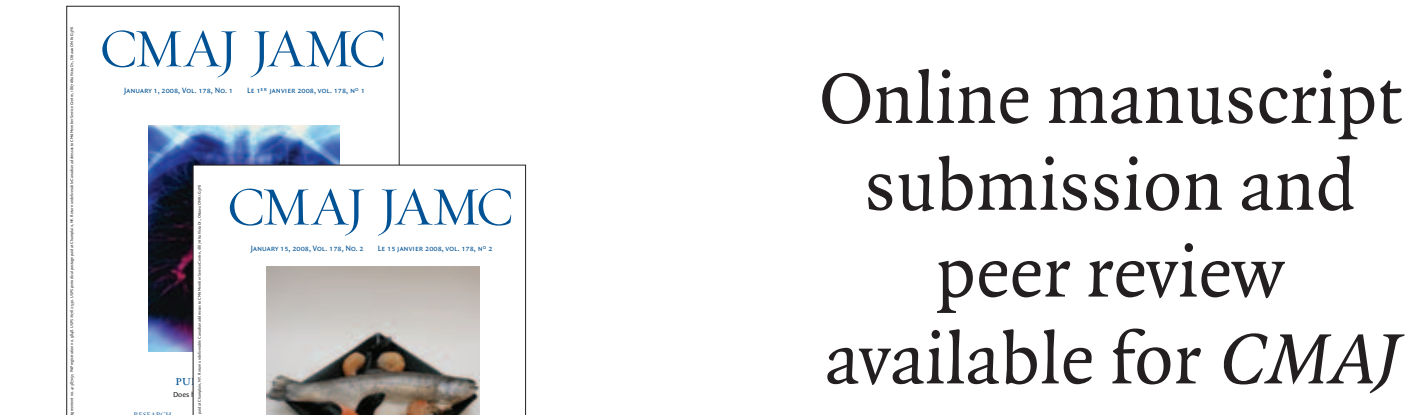

http://mc.manuscriptcentral.com/cmaj 